\title{
Microspray and microflow liquid chromatography: the way forward for LC-MS bioanalysis
}

\author{
Shane R Needham*,1 \\ ${ }^{1}$ Alturas Analytics, Inc. 1324 Alturas Drive Moscow, ID 83843, USA \\ * Author for correspondence: sneedham@alturasanalytics.com
'The way forward for bioanalytical MS is MFLC-MS, yet bioanalysts today may not even recognize the look of the fully 'integrated' instrument 20 years from now."

First draft submitted: 29 September 2017; Accepted for publication: 5 October 2017; Published online: 5 December 2017

Keywords: bioanalysis $\bullet$ LC-MS • LC-MS/MS • microflow • microspray • nanospray

It is well known that the efficiency of ionization improves as the liquid flow rate to the source decreases for ESI. The improved ionization efficiency leads to better MS signal [1]. Microflow liquid chromatography (MFLC) has also been shown to be advantageous for over 40 years [2]. Conventional LC-MS flow rates are in the range of 250-600 $\mathrm{\mu l} / \mathrm{min}$ with column internal diameters of $2.1 \mathrm{~mm}$. Nanospray, a technique highly developed by Gary Valaskovic utilizes flow rates in the order of sub $\mu \mathrm{l} / \mathrm{min}$ with column internal diameters of $<100 \mu \mathrm{m}$ [3]. Microspray (also known as 'high flow nanospray') is a technique that typically consists of flow rates of 5-100 $\mu \mathrm{l} / \mathrm{min}$ with column diameters from $0.25-1.0 \mathrm{~mm}$. Microspray and MFLC combined, are a great means to achieve the benefits of improved MS signal from nanospray and optimal analysis times shown by conventional LC-MS flow rates [4,5].

\section{The 'sampling advantage' of MFLC-MS}

The benefits of MFLC-MS include improved signal, low solvent consumption, reduced matrix effects and reduced source contamination. However the biggest benefit in using MFLC-MS/MS in bioanalysis is what we like to call the effective 'sampling advantage' [5]. The improved signal afforded by MFLC-MS compared with conventional LC-MS allows the bioanalyst to inject less sample and achieve the same signal as conventional LC-MS. As more assays transition to microsampling and micro volumes, the bioanalyst relies on a smaller sample size for a more conveniently collected and/or more concentrated sample [6]. As an example, if the bioanalyst has $100 \mu \mathrm{l}$ of final sample volume and uses conventional LC $(2.1 \mathrm{~mm}$ ID column), the injection volume could likely be $40 \mu \mathrm{l}$ to achieve the desired MS signal. In comparison with MFLC, the bioanalyst could use an injection volume of 1-5 $\mu$ l and achieve similar MS signal from the original final sample volume. In our laboratory, our bioanalysts call this a 'peace of mind', since many (>10) injections could be made from the same sample with minimal concern of running out of sample. In the bioanalytical world, equipment failures, misinjections, stopped runs and other issues are not desired but expected to happen. Thus, the ability for many injections from the same sample gives the bioanalyst a 'peace of mind' to think that 'if all else fails, I can re-inject my sample(s).' For any scientist that has analyzed samples over-night only to arrive in the morning to see there was a misinjection, this convenience (ability to inject numerous samples) is invaluable and is the true high performance of MFLC-MS.

The reason to implement MFLC are as compelling as the motivation to switch from $4.6 \mathrm{~mm}$ ID columns (common in LC-MS in the early 1990s) to $2.1 \mathrm{~mm}$ ID columns. With the effective 'sampling advantage', it is difficult to imagine bioanalytical laboratories returning to $4.6 \mathrm{~mm}$ ID columns at $1-2 \mathrm{ml} / \mathrm{min}$ flowrate [7].

\section{System volume challenges}

As column volumes are miniaturized, attention to the overall plumbing and connection scheme, starting at the outlet of the HPLC pump is required. An MFLC column of dimensions, $0.3 \mathrm{~mm}$ ID $\times 50 \mathrm{~mm}$ in length has an 
approximate total column volume of $3 \mu$ l. Thus, total system volume, the injection volume, as well as pre- and postcolumn volume is critical for the efficiency of the chromatographic peaks and optimal run times. To minimize the overall run time and the gradient delay from the LC pump, the precolumn volume (tubing connections with pump, volume through autosampler and connections to the column) should be reduced as much as feasible. In order to maintain efficient and symmetrical peaks, the postcolumn connections to the ESI source should be minimized as much as possible. Integration of the LC column into the source of the ESI emitter provides such a benefit. In fact, a packed emitter column called PicoFrit ${ }^{\text {TM }}$ has been used in proteomics for the past 20 years [8]. Recently, integrated columns have shown utility in bioanalysis $[9,10]$. Integrated columns in bioanalysis yielded improved chromatographic peak shape and improved signal to noise compared with conventional LC columns.

\section{Barriers to adoption of MFLC-MS}

A discussion of the challenges of the wide spread implementation of MFLC-MS is only fair with respect to science. As with any technology, the widespread adoption typically lies with the end user (bioanalyst) not being familiar with or comfortable with the technology. I think there are two reasons that have slowed the wide spread implementation of MFLC-MS. Reason number one is mostly user based hesitation and the other is the apparent challenge coupling a miniaturized system (minimal system dwell volumes required) with the MS. Both of these challenges lead to an inconvenient experience by the user and both challenges can be solved with a good collaboration of users and instrument manufacturers.

The first reason/or end user dilemma is that current liquid flow pumps are only capable of one mode (MFLC or conventional HPLC) of flow rate operation. Thus, the bioanalyst is required to change from one pump to the other when conventional and MFLC methods are developed on the same mass spectrometer, which is common practice in the bioanalytical laboratory. Even if the change-over process is a few minutes, this is still a loss of productivity and the process is difficult to perform on an unattended run overnight. The second reason for widespread adoption of the technology is the inefficient coupling of MFLC with the MS. Where MFLC columns can have total column volumes of $2-10 \mu \mathrm{l}$, the proximity of the MFLC column to the autosampler, HPLC pumps and MS requires excessive tubing and connections to the MS interface. Current set-up of MFLC-MS instruments may have extra column dwell volumes greater than the column volume. This leads to poor chromatographic peak shapes and suboptimal run times. Both of these challenges are not insurmountable and with patience and collaboration (instrument manufacturers and users) new technology will be developed for a liquid flow pump to deliver sub $\mu \mathrm{l} / \mathrm{min}$ to $\mathrm{ml} / \mathrm{min}$ flow rates. Manufacturers will also optimize instruments where the LC column is integrated into the source. The integrated MFLC-MS of the future with the autosampler, LC and MS could even look like one encompassed unit with no external connections. These changes will minimize system dwell volumes; will expand the user base and acceptance of MFLC-MS as routine.

\section{Conclusion}

MFLC-MS is convenient (it works) and this truly is the high performance of MFLC-MS. The acceptance of MFLC-MS will continue to grow. Liquid flow pumps will be developed that handle flow rates for MFLC and HPLC in the same unit. Integrated technologies will be developed to interface autosamplers, MFLC and MS to minimize connections and dwell volumes. Especially as more large molecules are developed as therapeutics, MFLC-MS will be the perfect fit. The way forward for bioanalytical MS is MFLC-MS, yet bioanalysts today may not even recognize the look of the fully 'integrated' instrument 20 years from now.

\section{Acknowledgements}

A special thanks to $G$ Valaskovic of New Objective and MS Lee of Milestone Development Services for insightful discussions. The author also like to thank C. Christianson and A Hoffman of Alturas Analytics, Inc. for their vision for the future of MFLC-MS.

Financial \& competing interests disclosure

The author has no relevant affiliations or financial involvement with any organization or entity with a financial interest in or financial conflict with the subject matter or materials discussed in the manuscript. This includes employment, consultancies, honoraria, stock ownership or options, expert testimony, grants or patents received or pending, or royalties.

No writing assistance was utilized in the production of this manuscript. 


\section{References}

1. Kebarle P, Tang L. From ions in solution to ions in the gas phase: the mechanism of electrospray ionization mass spectrometry. Anal. Chem. 65, A972-A986 (1993).

2. Ishii D, Asai K, Hibi K, Jonokuchi T, Nagaya M. A study of micro-high-performance liquid chromatography. J. Chromatogr. A 144(2), 157-168 (1977).

3. Valaskovic GA, Utley L, Lee MS, Wu JT. Ultra-low flow nanospray for the normalization of conventional liquid chromatography/mass spectrometry through equimolar response: standard-free quantitative estimation of metabolite levels in drug discovery. Rapid Comm. Mass Spec. 20(7), 1087-1096 (2006).

4. Johnson CJL, Christianson CD, Needham SR. The advantages of microflow LC-MS/MS compared with conventional HPLC-MS/MS for the analysis of methotrexate from human plasma. Bioanalysis 5(11), 1387-1396 (2013).

5. Needham SR, Valaskovic G. Peptide and protein bioanalysis using integrated column-to-source technology for high-flow nanospray. Chapter 5. In: Protein Analysis Using Mass Spectrometry: Accelerating Protein Biotherapeutics from Lab to Patient. Lee MS, Li QC (Eds). John Wiley and Sons, Inc. (2017)

6. Stove C, Spooner N. DBS and beyond. Bioanalysis 7(16), 1961-1962 (2015).

7. Covey TE, Henion J. High-speed liquid chromatography/tandem mass spectrometry for the determination of drugs in biological samples. Anal Chem. 58, 2453-2460 (1986).

8. Emmett M, Caprioli R. Microspray-electrospray mass spectrometry: ultra-high-sensitivity analysis of peptides and proteins. J. Am. Soc. Mass Spectrom. 5(7), 605-613 (1994).

9. Johnson CC, DeChenne CS, Needham S, Valaskovic G. Validation of an in-source micro flow LC-MS/MS method for bioanalysis. Presented at: The 62nd Conference on American Society for Mass Spectrometry. MD, USA, 15-19 June 2014.

10. Kleinnijenhuis A, Ingola M, Toersche J, van Holthoon F, van Dongen W. Quantitative bottom up analysis of infliximab in serum using protein A purification and integrated LC-electrospray chip IonKey MS/MS technology. Bioanalysis 8(9), 891-904 (2016). 
